\title{
Review Article \\ Electrically Tuned Microwave Devices Using Liquid Crystal Technology
}

\author{
Pouria Yaghmaee, ${ }^{1}$ Onur Hamza Karabey, ${ }^{2}$ Bevan Bates, ${ }^{1,3}$ \\ Christophe Fumeaux, ${ }^{1}$ and Rolf Jakoby ${ }^{2}$

\footnotetext{
${ }^{1}$ School of Electrical and Electronic Engineering, University of Adelaide, Adelaide, SA 5005, Australia

${ }^{2}$ Institute for Microwave Engineering and Photonics, Technische Universität Darmstadt, 64283 Darmstadt, Germany
} \\ ${ }^{3}$ Defence Science and Technology Organisation, Edinburgh, SA 5111, Australia
}

Correspondence should be addressed to Pouria Yaghmaee; pouria@eleceng.adelaide.edu.au

Received 16 July 2013; Accepted 7 September 2013

Academic Editor: Francisco Falcone Lanas

Copyright (C) 2013 Pouria Yaghmaee et al. This is an open access article distributed under the Creative Commons Attribution License, which permits unrestricted use, distribution, and reproduction in any medium, provided the original work is properly cited.

\begin{abstract}
An overview of liquid crystal technology for microwave and millimeter-wave frequencies is presented. The potential of liquid crystals as reconfigurable materials arises from their ability for continuous tuning with low power consumption, transparency, and possible integration with printed and flexible circuit technologies. This paper describes physical theory and fundamental electrical properties arising from the anisotropy of liquid crystals and overviews selected realized liquid crystal devices, throughout four main categories: resonators and filters, phase shifters and delay lines, antennas, and, finally, frequency-selective surfaces and metamaterials.
\end{abstract}

\section{Introduction}

As a result of rapid growth in wireless communications systems, much attention has been devoted to designing and manufacturing frequency tunable devices, for example, resonators, filters, antennas, and frequency-selective surfaces [1]. The increased demand on the functionality and performance of portable wireless devices and the scarcity of frequency spectrum stimulate the need for designing frequency tunable and flexible microwave and millimeter-wave devices. In the past, various different tuning methods have been discussed for wireless radio frequency (RF) and microwave devices. These frequency tuning methods can be classified into two main types, either discrete or continuous. In the discrete case, frequency tuning is a switching mechanism for operation at distinct fixed frequencies, thus resulting in incomplete frequency coverage.

In contrast, continuous frequency tuning allows for smooth transition between operating bands without skipping over frequencies.

The actual physical processes that can cause frequency tuning can be grouped into three main categories: (a) mechanical actuation, (b) integrated electronic devices, and (c) tunable materials [1-3]. The most common and practical techniques used for achieving mechanical actuations are electromechanical, piezoelectric, hydraulic/pneumatic and microactuations such as microelectromechanical systems (MEMS) [4-8]. Integrated electronic tunable devices on the other hand can be achieved through using PIN diodes, MEMS switches, field-effect transistors (FETs) switches, optoelectronic switches, and varactor diodes [9-13]. The main drawbacks of these switches at microwave and millimeter-wave frequencies are their discrete tuning and/or their low quality factor, especially at higher frequencies and high fabrication effort with low yield.

The third method for attaining tuning is using tunable materials. In this case, the permittivity and permeability of a particular material can be tuned through an external field, either electric or magnetic, typically enabling continuous frequency tuning. The most frequently used tunable materials are ferrites, ferroelectrics, and semiconductors.

A further specific kind of tunable materials, namely, liquid crystals, has been recently presented in the literature for microwave and millimeter-wave applications. Liquid crystals 
are promising tunable microwave materials especially at frequencies above $10 \mathrm{GHz}$ (due to lower losses at higher microwave frequencies), as they are flexible and continuously tunable. Additionally, they require a low bias voltage and satisfy low dielectric constant requirements [14-16]. In the following, the properties and characteristics of liquid crystal technology for use in the microwave range are explained and discussed, followed by an overview of the various practical realizations of microwave and millimeter-wave devices proposed in the literature.

\section{Liquid Crystals}

Liquid crystals are dielectric materials with anisotropic characteristics, which can be grouped into three different mesophases between solid/crystalline and liquid/isotropic states. These mesophases can be separated by orientation and positional order into nematic, smetic, and cholesteric [17]. The nematic phase is the most commonly used phase of liquid crystals at microwave and millimeter-wave frequencies and is characterized by an orientation of rod-shaped molecules resulting in a highly anisotropic permittivity tensor. In this phase, the preferential, that is, the average direction of the molecular axes, is described by the director, denoted here as $\vec{n}$, which is aligned with the long axis of the rod-shaped liquid crystal molecules.

2.1. Anisotropy States. The anisotropy tensor exhibits a relative permittivity $\varepsilon_{\|}$in the director direction $\vec{n}$ and $\varepsilon_{\perp}$ in orthogonal directions [18-20]. In a typical capacitive arrangement, where a liquid crystal cell is sandwiched between two metal electrodes and no bias voltage is applied $\left(V_{b}=\right.$ $0 \mathrm{~V}$ ), the initial alignment of the liquid crystal molecules is achieved through coating the boundary surfaces (preferably the top and bottom layer of the cell) with a thin layer of polyimide film and then mechanically rubbing them using a velvet cloth. The rubbing creates microscopic grooves in the polyimide surfaces (Figure 1) and enables preferred alignment for the liquid crystal molecules in the unbiased state [21, 22] (Figures 2(a) and 2(b)). In electromagnetic simulations, these extremely thin polyimide layers can be neglected to a good approximation.

In this initial case $\left(V_{b}=0 \mathrm{~V}\right)$, the director $\vec{n}$ will be parallel to the metallic layers and the relative permittivity tensor relevant for the interlayer material is defined as

$$
\overleftrightarrow{\varepsilon_{\perp}}=\left(\begin{array}{ccc}
\varepsilon_{\|} & 0 & 0 \\
0 & \varepsilon_{\perp} & 0 \\
0 & 0 & \varepsilon_{\perp}
\end{array}\right)
$$

For this case, the scalar effective relative permittivity determining the capacitance can be approximated as $\varepsilon_{\text {eff }}=$ ${\overleftrightarrow{\varepsilon_{\perp}(z, z)}}=\varepsilon_{\perp}$, since the molecules orient along the $z$-axis and provide electric field in the $z$-direction. This is commonly known as the perpendicular state (Figure 2(b)).

As bias voltage $V_{b}$ starts to increase $\left(V_{\text {th }}<V_{b}<V_{\max }\right)$, the orientation of the director $\vec{n}$ continuously changes from the perpendicular to the parallel state [23]. The random

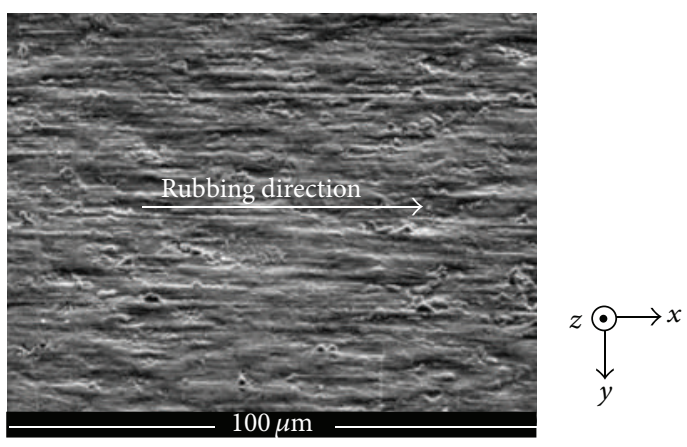

FIGURE 1: Scanning electron microscope image of a copper patch, coated with a $0.3-0.4 \mu \mathrm{m}$ thick of polyimide film and mechanically rubbed, along the $x$-axis layer.

distribution of the liquid crystal molecules in this case can be described by the order parameter $S$, expressed by

$$
S=\left\langle\frac{3}{2} \cos ^{2} \theta-\frac{1}{2}\right\rangle,
$$

where in this equation $\theta$ is the average angle between the molecular axis and the director $\vec{n}$ (average direction in a volume element of a liquid crystal sample) and $<>$ corresponds to the mathematical expectation operator, averaging the orientation of all molecules [24, 25].

The difference between the perpendicular and parallel state is identified as the dielectric anisotropy $\Delta \varepsilon$ of the liquid crystal molecules and can be expressed as

$$
\Delta \varepsilon=\varepsilon_{\|}-\varepsilon_{\perp}
$$

By taking into account $\Delta \varepsilon$ and defining $\otimes$ as the tensor product of two vectors, the permittivity tensor for the intermediate state can be described as $[23,26]$

$$
\begin{aligned}
\overleftrightarrow{\varepsilon} & =\varepsilon_{\perp} \cdot \overleftrightarrow{1}+\Delta \varepsilon \cdot(\vec{n} \otimes \vec{n}) \\
& =\varepsilon_{\perp} \cdot\left(\begin{array}{lll}
1 & 0 & 0 \\
0 & 1 & 0 \\
0 & 0 & 1
\end{array}\right)+\Delta \varepsilon \cdot\left(\begin{array}{ccc}
n_{x}^{2} & n_{x} n_{y} & n_{x} n_{z} \\
n_{x} n_{y} & n_{y}^{2} & n_{y} n_{z} \\
n_{x} n_{z} & n_{y} n_{z} & n_{z}^{2}
\end{array}\right)
\end{aligned}
$$

which can be expressed as

$$
\overleftrightarrow{\varepsilon}_{i}=\left(\begin{array}{ccc}
\varepsilon_{\perp}+\Delta \varepsilon \sin ^{2} \theta & 0 & \Delta \varepsilon \sin \theta \cos \theta \\
0 & \varepsilon_{\perp} & 0 \\
\Delta \varepsilon \sin \theta \cos \theta & 0 & \varepsilon_{\perp}+\Delta \varepsilon \cos ^{2} \theta
\end{array}\right)
$$

for the case when the rubbing direction of the polyimide is along the $x$-axis (see Figures 1 and $2(\mathrm{c})$ ). The effective relative permittivity in this case becomes $\varepsilon_{\text {eff }}=\overleftrightarrow{\varepsilon}_{i(z, z)}=\varepsilon_{i}$, due to the E-field along the $z$-axis.

As the bias voltage increases to a certain voltage threshold $\left(V_{b} \geq V_{\max }\right)$, the liquid crystal molecules become stable. In this state, the relevant effective permittivity ( $\left.\varepsilon_{\text {eff }}\right)$ gradually 


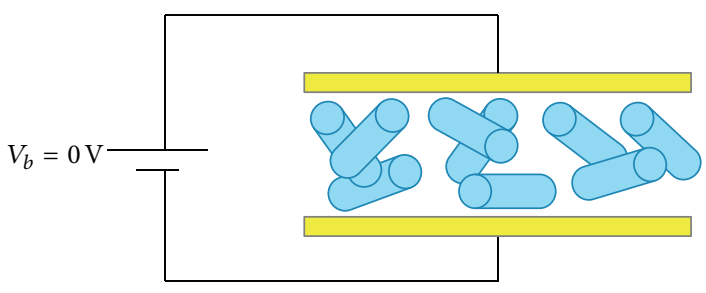

(a)

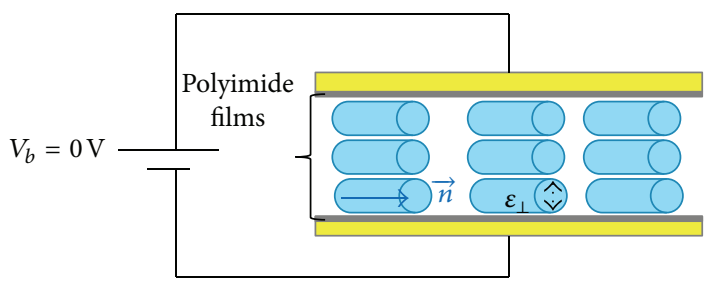

(b)

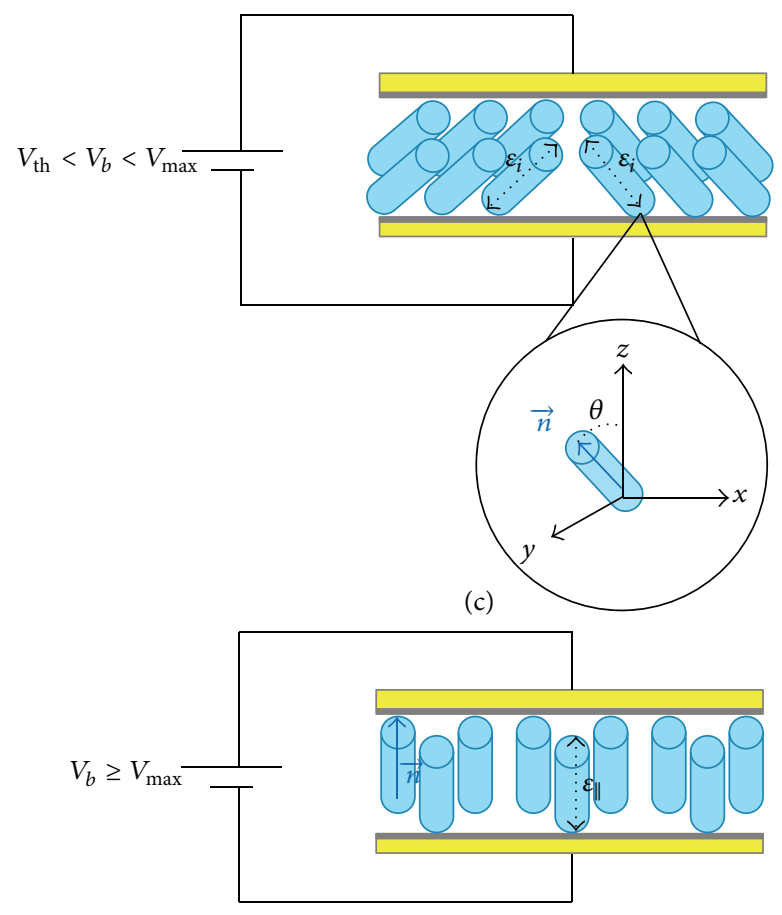

(d)

FIGURE 2: Liquid crystal molecule orientation in different states (a) no-polyimide films, random orientation, (b) polyimide films, perpendicular state $\left(\varepsilon_{\text {eff }}=\varepsilon_{\perp}\right)$, (c) intermediate state $\left(\varepsilon_{\text {eff }}=\varepsilon_{i}\right)$, and (d) parallel state $\left(\varepsilon_{\text {eff }}=\varepsilon_{\|}\right)$.

aligns along the director $\vec{n}$, which results in the permittivity tensor becoming

$$
\overleftrightarrow{\varepsilon_{\|}}=\left(\begin{array}{ccc}
\varepsilon_{\perp} & 0 & 0 \\
0 & \varepsilon_{\perp} & 0 \\
0 & 0 & \varepsilon_{\|}
\end{array}\right) .
$$

This tensor describes a full alignment of the liquid crystal directors along the static electric field lines associated with the applied voltage. The effective relative permittivity in this case becomes $\varepsilon_{\text {eff }}=\overleftrightarrow{\mathcal{E}}_{\|(z, z)}=\varepsilon_{\|}$, given that the liquid crystal molecules orient along the $z$-axis and provide electric field along the $z$-direction. This is generally referred to as

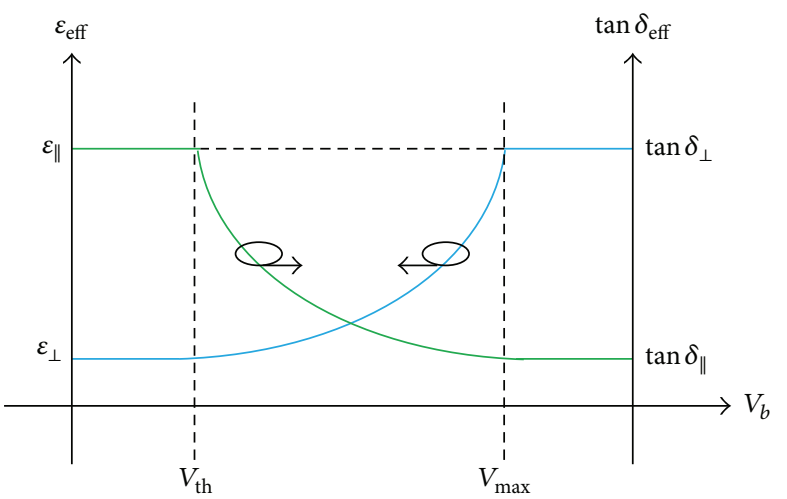

FIGURE 3: Effective relative permittivity of perpendicular $\left(\varepsilon_{\perp}\right)$ and parallel $\left(\varepsilon_{\|}\right)$states and loss tangent $\left(\tan \delta_{\perp}\right.$ and $\left.\tan \delta_{\|}\right)$as function of the applied bias voltage $\left(V_{b}\right)$.

the parallel state, that is, the bias electric field point parallel to $\vec{n}$, Figure $2(\mathrm{~d})$.

From the description above, it becomes clear that the biasdependent transition from perpendicular to parallel state provides a continuous variation of the effective permittivity between perpendicular to parallel states.

The material tuning commonly referred to as frequency tuning/frequency variation/tunability $(\tau)$ can be expressed as a function of the highest and lowest resonant frequencies $f_{h}$ and $f_{l}$ as

$$
\tau=\left(\frac{f_{h}-f_{l}}{f_{h}}\right) 100 \%
$$

or

$$
\tau=\left(\frac{f_{h}-f_{l}}{f_{l}}\right) 100 \%
$$

depending on which frequency is chosen as a reference. Alternatively, the tunable range/tuning range $(\tau R)$ caused by this variation can also be expressed as $[1,19]$

$$
\tau R=\frac{2\left(f_{h}-f_{l}\right)}{\left(f_{h}+f_{l}\right)} 100 \% .
$$

Figure 3 illustrates the evolution of the relative permittivity and the equivalent loss tangent between the two extreme states (perpendicular and parallel) when the bias voltage $V_{b}$ is increased from the threshold voltage $V_{\text {th }}$ to the saturation voltage $V_{\max }$.

For available liquid crystal mixtures, the typical relative permittivity value for the perpendicular $\left(\varepsilon_{\perp}\right)$ state is around 2.2 to 2.8 , while for the parallel $\left(\varepsilon_{\|}\right)$state is between 2.6 and 3.5. The loss tangents have typical values of $\tan \delta \leq$ 0.02 for both states. These insertion losses depend on the topology, material, and operational frequency [18, 27]. Table 1 lists the properties of some of the most common liquid crystal (nematic) samples used at microwave and millimeter-wave frequencies. The listed values might slightly vary, depending on the working frequency and room temperature.

2.2. Electromagnetic Simulation Modelling. Liquid crystals are generally modelled in electromagnetic simulation 
TABLE 1: Properties of liquid crystals at $20^{\circ} \mathrm{C}$ temperature, as specified at the given operating frequency.

\begin{tabular}{lccccccc}
\hline \multirow{2}{*}{ Samples } & \multicolumn{2}{c}{ Permittivity } & Dielectric anisotropy & \multicolumn{2}{c}{ Loss tangent } & \multicolumn{2}{c}{ Operating frequency } \\
& $\varepsilon_{\perp}$ & $\varepsilon_{\|}$ & $\Delta \varepsilon$ & $\tan \delta_{\perp}$ & $\tan \delta_{\|}$ & $F(\mathrm{GHz})$ \\
\hline K15 (5CB) & 2.72 & 2.90 & 0.18 & 0.03 & 0.03 & $1-10$ & {$[28-31]$} \\
BL037 & 2.35 & 2.61 & 0.26 & 0.06 & 0.06 & {$[-10$} & {$[28,41]$} \\
BL006 & $2.62-2.69$ & $3.11-3.12$ & $0.49-0.43$ & $0.015-0.007$ & $0.035-0.036$ & $4.8-8.7$ & {$[16]$} \\
E7 & 2.72 & 3.17 & 0.45 & 0.12 & 0.02 & $5-6$ & $13.6-19$ \\
GT3-23001 & $2.46-2.50$ & $3.28-3.30$ & $0.82-0.80$ & 0.0143 & $0.0038-0.004$ & {$[14,54,60]$} \\
GT3-24002 & 2.50 & 3.30 & 0.80 & 0.0123 & 0.0032 & $6-10$ & {$[62]$} \\
\hline
\end{tabular}

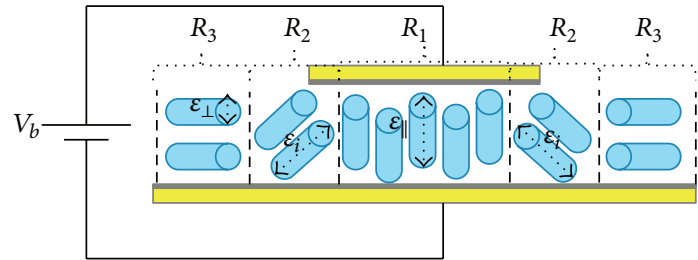

FIGURE 4: Liquid crystal cell regions for $V_{b}=V_{\max }$, with region $R_{1}$ in the center, simulated as the parallel state, region $R_{2}$ as the intermediate state, and region $R_{3}$ on both sides simulated as the perpendicular state.

software tools as a material with defined permittivity and loss tangent, which can vary depending on the biasing state of the liquid crystal molecules. With few exceptions, electromagnetic full-wave simulation tools only allow simulation of anisotropic materials with the director $\vec{n}$ along the principal directions (perpendicular and parallel), as formulated in (1) and (6). These simple anisotropy matrices allow the approximate simulation of the unbiased and saturated bias cases, where all the molecules are aligned along one of the principal directions. However, the static biasing fields will, in most cases, exhibit fringing fields with components not aligned to one of the principal axis. To reduce the error associated with the assumption that all fields are aligned along principal axes, the liquid crystal cell can be divided into three main regions $\left(R_{1}, R_{2}\right.$, and $\left.R_{3}\right)$, as proposed in [26] and shown in Figure 4.

Through this simulation method, the initial region $\left(R_{1}\right)$ is calculated using (6), given that the biasing electric field $\vec{E}$ in this region is mainly parallel to the director $\vec{n},\left(\varepsilon_{\text {eff }}=\right.$ $\left.\varepsilon_{\|}\right)$. However, in the second region $\left(R_{2}\right)$, due to the fact that the liquid crystal molecules in this region are neither perpendicular nor parallel, the effective permittivity $\varepsilon_{\text {eff }}$ in this case is calculated from (5), with effective permittivity approximated as $\varepsilon_{i}$ for an angle $\theta=45$ degrees.

Finally, the side regions $\left(R_{3}\right)$ can also be simulated by (1), given that the bias voltage does not affect this region significantly, and thus the director $\vec{n}$ of the molecules is pointed in the perpendicular state $\left(\varepsilon_{\mathrm{eff}}=\varepsilon_{\perp}\right)$.

\section{Applications}

Owing to the anisotropic properties of liquid crystal compounds and mixtures, there are a number of reported devices

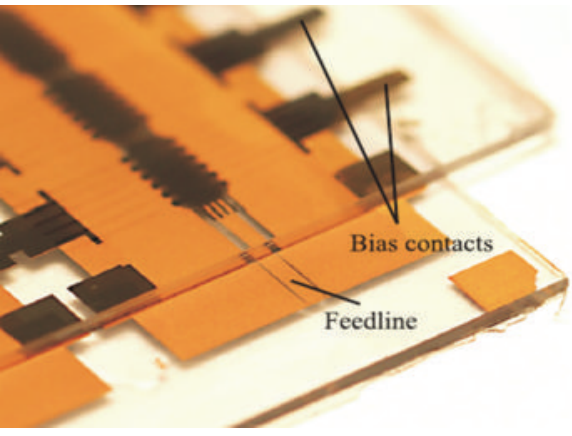

FIGURE 5: Photograph of the realized tunable filter containing liquid crystal mixture [32].

which use liquid crystal properties to achieve electrical tuning. The description of these devices is divided into four main categories: (1) resonators and filters, (2) phase shifters and delay lines, (3) antennas, and (4) frequency-selective surfaces and metamaterials. In the next section, liquid crystal tunable devices designed for operation at microwave and millimeter-wave frequencies are discussed.

3.1. Resonators and Filters. Tunable resonators and filters that exploit the dielectric anisotropy of liquid crystal have been discussed in the literature. One of the earliest examples of liquid crystal resonators is reported in [28]. The study illustrated a half-wavelength open-circuited stub resonator and a second-order dual behaviour resonator filter. Using two standard liquid crystal samples (K15 and BL037 [2831]), frequency shifts of a few percent for lower microwave frequencies (below X-band) have been achieved.

In a different example, a liquid crystal tunable band-pass filter was presented [32] (Figure 5). The proposed three-pole filter operates at a center frequency of around $20 \mathrm{GHz}$ and demonstrates approximately $2 \mathrm{GHz}$ of frequency shifting. A comparable tunable coupled microstrip line filter has been developed with an operational frequency of $33 \mathrm{GHz}$ [33]. The E7 liquid crystal sample employed in this structure demonstrates tunability of up to $2 \mathrm{GHz}$.

Even though liquid crystals have primarily been discussed for high microwave and millimeter-wave electromagnetic structures, a recent study has investigated the potential of these materials for even lower microwave frequency ranges even down to S-band. A tunable S-band resonator with an operational frequency of $3.5 \mathrm{GHz}$ was presented in 


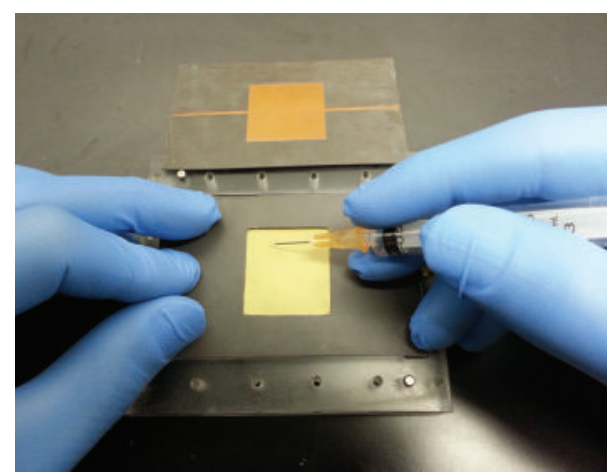

FIGURE 6: Fabricated S-band resonator, during liquid crystal (GT323001) filling process [14].

$[14,34]$ (Figure 6). The two sandwiched liquid crystal samples (K15 and GT3-23001) in this resonator allowed for $4 \%$ and $8 \%$ tuning range, respectively. The work suggests possible application of liquid crystal technology in future designs, for manufacturing microwave lower frequency devices for specific applications, for example, in conjunction with flexible electronics.

3.2. Phase Shifters and Delay Lines. Apart from the resonators and filters, liquid crystals have been demonstrated in the design of phase shifters and delay lines. An early example of phase shifters is reported in [35], where a voltage-controlled $20^{\circ}$ phase shift was achieved with a K15 sample at a center frequency of $10.5 \mathrm{GHz}$. Furthermore, in [36], a planar integrated tunable phase shifter has been presented. By filling the channels with liquid crystal and applying a bias voltage, a differential phase shift of $53^{\circ}$ was achieved at a center frequency at about $18 \mathrm{GHz}$.

In a different example, reducing the liquid crystal cell thickness was discussed as a technique for improving tuning/switching speed [37]. In this work, using only $5 \mu \mathrm{m}$ thickness of liquid crystals instead of the more common $500 \mu \mathrm{m}$ resulted in $60^{\circ}$ of phase shift with a tuning speed of $340 \mathrm{~ms}$. Dual-frequency switching mode was proposed for active alignment of the liquid crystal molecules in two principal directions [38]. By controlling the LC molecule orientation, lower insertion loss and faster response times were observed in the phase shift of a microwave variable delay line. A microwave variable delay line using a membrane impregnated with liquid crystal has also been fabricated [39]. Experimental results indicated a $270^{\circ}$ phase shift for the variable delay lines with a tuning response time of around $33 \mathrm{~ms}$.

In a more recent case, a $2 \mathrm{D}$ electronically-steered phased array with a variable delay line was presented [40]. The $17.5 \mathrm{GHz}$ phased array demonstrated a maximum differential phase shift of $300^{\circ}$ (Figure 7).

3.3. Antennas. Electrically tuned liquid crystal antennas have been widely discussed in recent years. The key in these designs is to form a sandwich-shaped structure, with a metal patch on top, a ground plane at the bottom of the structure,

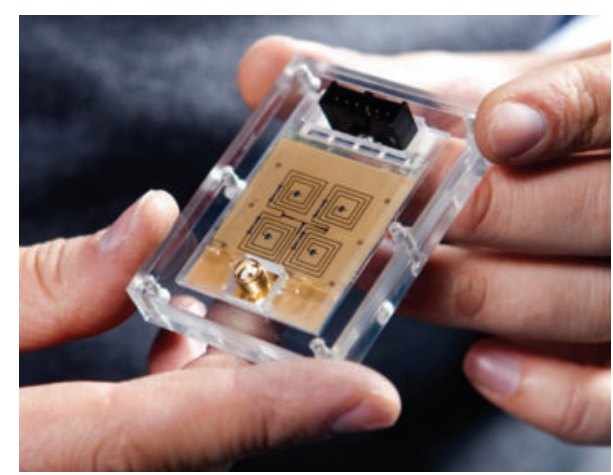

FIGURE 7: Prototype of the electronic beam steering liquid crystal phased array [40].

and liquid crystal in between in a central cell. The patterned patch and ground planes are used both for radiation and as electrodes in the biasing circuit. Liquid crystal antennas can be divided into three main categories: (1) frequency tunable antennas, (2) beam steering antennas and reflectarrays, and (3) polarization agile antennas. In the following, each of these categories are discussed separately.

3.3.1. Frequency Tunable Antennas. One of the initially reported tunable antennas had liquid crystal placed inside its intermediate foam substrate, underneath the patch [41]. Upon application of a varying external bias voltage to this structure, a frequency shift of $140 \mathrm{MHz}$ was obtained around the frequency of 4.74 and $4.6 \mathrm{GHz}$, which corresponds to $2.95 \%$ frequency variation.

A comparable rectangular patch demonstrated a 5.5\% tunability with respect to lower frequency [42], in a similar frequency range $(\sim 4.5 \mathrm{GHz})$. For these two antennas, standard commercial K15 and BL037 liquid crystal samples were used. Similarly, a sandwich structure antenna operating at a frequency of $5 \mathrm{GHz}$ was presented in [43]. This antenna was designed using three layers of Taconic glass-reinforced PTFE substrate placed on top of each other, with E7 liquid crystal in its central layer. The simulation of this arrangement predicted a tuning range of $8 \%$, whereas measurement demonstrated a tuning range of $4 \%$.

Liquid crystal has in addition been demonstrated in combination with newly developed flexible materials. An example of a tunable multilayer patch antenna on flexible liquid crystal polymer (LCP) substrates is reported in [44]. This work shows that by using novel high-performance liquid crystal mixtures, a continuous frequency tuning range of $10 \%$ at Ka band $(\sim 35 \mathrm{GHz})$ was achieved through application of an external bias voltage.

3.3.2. Beam Steering Antennas and Reflectarrays. The use of liquid crystal has been reported for the development of leaky-wave antennas able to steer their main beam direction at a fixed frequency. In [45], a leaky-wave antenna based on composite right/left-handed waveguide was described, with beam steering at $7.6 \mathrm{GHz}$. Through application of either 
a static electric or magnetic field to the liquid crystal molecules, a beam tilt of around $\pm 10^{\circ}$ was achieved.

Apart from phased arrays, reconfigurable reflectarrays could benefit greatly from the application of liquid crystals in their tuning mechanism. One of the first reflectarrays with liquid crystal tuning is reported in [46, 47]. In this design, a liquid crystal tunable reflectarray was presented and verified. Measurements demonstrated $300^{\circ}$ of phase tunability of a unit cell, through applying bias voltages up to $40 \mathrm{~V}$. A comparable example was reported in [29]. In this work, numerical and measured results demonstrated $180^{\circ}$ of tunable phase shift at X-band using K15 liquid crystal. In a similar frequency range, a reflectarray antenna was presented using a BL006 sample $[16,48]$. The electronic tuning of the effective permittivity of the liquid crystal in this study [16] demonstrated reconfiguration of monopulse sum and difference patterns.

Liquid crystals have also been demonstrated for reflectarrays at higher microwave frequencies, even above X-band. Due to the decreasing liquid crystal loss tangent $\delta$ with increasing frequency more attention has been directed in recent years towards designing millimeter-wave reflectarray cells. A recent reflectarray designed for operational frequencies of $77 \mathrm{GHz}$ suggests the possibility of $280^{\circ}$ element phase tunability through application of an external bias voltage [49]. Moreover in [50], a tuning phase range of $360^{\circ}$ was achieved for a reflectarray operating in a frequency range from 30 to $40 \mathrm{GHz}$. In a recent study a millimeter-wave reflectarray (F-band), which consists of $52 \times 54$ identical cells, has been designed to operate in the frequency range from 96 to $104 \mathrm{GHz}$ [51]. A GT3-23001 liquid crystal mixture was used to demonstrate the potential of the proposed reflectarray for beam scanning in F-band. This suggests that future wideband tunable antennas in the frequency range above $60 \mathrm{GHz}$ and even in the terahertz range up to $500 \mathrm{GHz}$ are possible. In similar concepts proposed in [52], phase changes of $165^{\circ}$ and $130^{\circ}$ were achieved for reflectarrays operating at a center frequency of 102 and $130 \mathrm{GHz}$, respectively.

3.3.3. Polarization Agile Antennas. In a recent work, polarization agile antennas using liquid crystal mixtures (GT323001) were reported in $[53,54]$ (Figure 8). This 2D beam steering phased array antenna consisted of a 2 by 2 dual-fed microstrip patch array and two separate feeding networks. Through reconfiguration of the feeding networks, dual linear and dual circular polarizations were achieved. Continuous tuning of the antenna polarization at a frequency of $13.75 \mathrm{GHz}$ was illustrated in this work for both simulation and prototype measurements.

3.4. Frequency-Selective Surfaces and Metamaterials. Other areas which have recently demonstrated high potential for tuning with liquid crystal materials are frequency-selective surface (FSS) and planar metamaterials. In these structures, the liquid crystal is generally sandwiched between two identical parallel substrates, consisting of printed patch patterns. Through application of an external bias voltage, a shift in the resonant frequency can be achieved. A concept of liquid

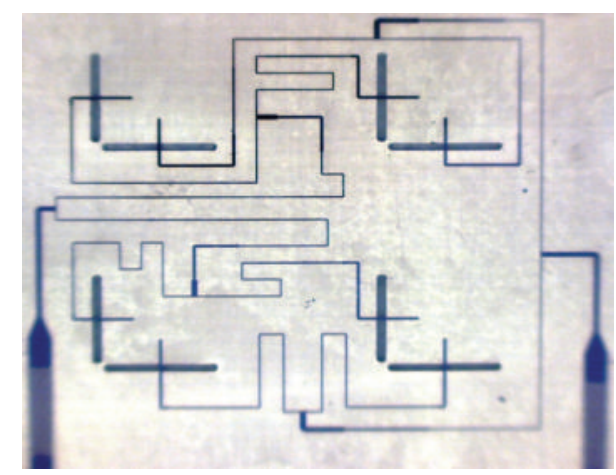

FIGURE 8: Prototype of a polarization agile antenna filled with liquid crystal mixture [54].

crystal tunable electric-LC (ELC) resonators is presented in [55], where tunability is achieved via using a microfluidic channel in the central capacitive gaps. The results achieved by simulation predicts $6 \%$ of continuous frequency tuning at around $4.5 \mathrm{GHz}$. The achieved results demonstrate the possibility of using these ELC resonators in an array to form a tunable FSS. An FSS with higher microwave frequency of 110-170 GHz (D-band) has been presented in [56]. The BL037 liquid crystal mixture in this device was sandwiched between two arrays of slot elements, illustrating a 3\% tunability. In another experimentally validated example, a magnetically tunable negative permeability metamaterial consisting of an array of broadside coupled split ring resonators (SRR) filled with liquid crystals has been proposed. Here a resonant frequency shift of $0.3 \mathrm{GHz}$ was obtained at X-band [57].

In other reported examples, perpendicular and parallel alignment of nematic liquid crystal cells have been demonstrated for developing metamaterial structures with index of refraction tunable from negative, through zero, to positive values [58-60]. Although some of the reported FSSs and metamaterial structures operate at higher frequencies (e.g., terahertz), the development of new liquid crystal mixtures suggests future potential applications at microwave and millimeter-wave frequencies.

\section{Summary}

Tunable devices operating at microwave and millimeterwave frequencies have been developed for many years. As part of these efforts, materials research has introduced a new reconfiguration approach through tunable liquid crystal mixtures. The need for flexibility and continuous tuning has opened a potential opportunity for the development of liquid crystal microwave technology. This has been recently exemplified with the emergence of newly developed liquid crystals mixtures specifically targeting this frequency range, such as GT3-23001 [14, 54, 61] and GT3-24002 [62]. Given that the effective permittivity of these anisotropic materials are voltage-dependent, liquid crystal could be ideal for various specific applications, for example, at millimeterwave frequencies where efficient tuning modalities are scarce or at microwave frequencies in conjunction with flexible electronics. 


\section{Conflict of Interests}

The authors declare that there is no conflict of interests regarding the publication of this paper.

\section{Acknowledgments}

The authors acknowledge A. Manabe from Merck KGaA, Darmstadt, Germany, and M. Nikfalazar from the Technische Universität Darmstadt, Germany, for their assistance and the Australian Research Council (ARC), under the Discovery Project DP120100661.

\section{References}

[1] A. Petosa, "An overview of tuning techniques for frequencyagile antennas," IEEE Antennas and Propagation Magazine, vol. 54, pp. 271-296, 2012.

[2] A. Petosa, "Frequency agile antennas for wireless communications-a survey," in Proceedings of the 14th International Symposium on Antenna Technology and Applied Electromagnetics and the American Electromagnetics Conference (ANTEM/AMEREM '10), pp. 1-4, Ottawa, Canada, July 2010.

[3] J. T. Bernhard, "Reconfigurable antennas," Synthesis Lectures on Antennas, vol. 2, pp. 1-66, 2007.

[4] G. H. Huff, D. L. Rolando, P. Walters, and J. McDonald, "A frequency reconfigurable dielectric resonator antenna using colloidal dispersions," IEEE Antennas and Wireless Propagation Letters, vol. 9, pp. 288-290, 2010.

[5] S. Hage-Ali, N. Tiercelin, P. Coquet, R. Sauleau, V. Preobrazhensky, and P. Pernod, "A millimeter-wave frequency tunable microstrip antenna on ultraflexible PDMS substrate," in Proceedings of the IEEE International Symposium on Antennas and Propagation and CNC-USNC/URSI Radio Science MeetingLeading the Wave, (AP-S/URSI '10), pp. 1-4, Toronto, Canada, July 2010.

[6] I. E. Khodasevych, W. S. T. Rowe, and A. Mitchell, "Reconfigurable fishnet metamaterial using pneumatic actuation," Progress in Electromagnetics Research B, vol. 38, pp. 57-70, 2012.

[7] S. A. Bokhari, J.-F. Zürcher, J. R. Mosig, and F. E. Gardiol, "A small microstrip patch antenna with a convenient tuning option," IEEE Transactions on Antennas and Propagation, vol. 44, no. 11, pp. 1521-1528, 1996.

[8] J. T. Bernhard, E. Kiely, and G. Washington, "A smart mechanically actuated two-layer electromagnetically coupled microstrip antenna with variable frequency, bandwidth, and antenna gain," IEEE Transactions on Antennas and Propagation, vol. 49, no. 4, pp. 597-601, 2001.

[9] C. J. Panagamuwa, A. Chauraya, and J. C. Vardaxoglou, "Frequency and beam reconfigurable antenna using photoconducting switches," IEEE Transactions on Antennas and Propagation, vol. 54, no. 2, pp. 449-454, 2006.

[10] W.-B. Zheng, Q.-A. Huang, X.-P. Liao, and F.-X. Li, "RF MEMS membrane switches on GaAs substrates for x-band applications," Journal of Microelectromechanical Systems, vol. 14, no. 3, pp. 464-471, 2005.

[11] D. Peroulis, K. Sarabandi, and L. P. B. Katehi, "Design of reconfigurable slot antennas," IEEE Transactions on Antennas and Propagation, vol. 53, no. 2, pp. 645-654, 2005.
[12] S. Kawasaki and T. Itoh, "A slot antenna with electronically tunable length," in Proceedings of the Antennas and Propagation Society Symposium, pp. 130-133, June 1991.

[13] P. Bhartia and I. Bahl, "A frequency agile microstrip antenna," in Proceedings of the Antennas and Propagation Society International Symposium, vol. 20, pp. 304-307, May 1982.

[14] P. Yaghmaee, C. Fumeaux, B. Bates, A. Manabe, O. H. Karabey, and R. Jakoby, "Frequency tunable S-band resonator using nematic liquid crystal," Electronics Letters, vol. 48, no. 12, pp. 798-7800, 2012.

[15] R. Dickie, P. Baine, R. Cahill et al., "Electrical characterisation of liquid crystals at millimetre wavelengths using frequency selective surfaces," Electronics Letters, vol. 48, pp. 611-612, 2012.

[16] D. E. Schaub and D. R. Oliver, "A circular patch resonator for the measurement of microwave permittivity of nematic liquid crystal," IEEE Transactions on Microwave Theory and Techniques, vol. 59, no. 7, pp. 1855-1862, 2011.

[17] S. Mueller, A. Penirschke, C. Damm et al., "Broad-band microwave characterization of liquid crystals using a temperaturecontrolled coaxial transmission line," IEEE Transactions on Microwave Theory and Techniques, vol. 53, no. 6, pp. 1937-1945, 2005.

[18] A. Gaebler, F. Goelden, S. Mueller, and R. Jakoby, "Efficiency considerations of tuneable liquid crystal microwave devices," in Proceedings of the German Microwave Conference, pp. 1-4, 2008.

[19] S. Mueller, M. Koeberle, F. Goelden et al., "W-Band characterization of anisotropic Liquid Crystals at room temperature," in Proceedings of the 38th European Microwave Conference (EuMC '08), pp. 119-122, Amsterdam, The Netherlands, October 2008.

[20] K. Yoshino, M. Inoue, H. Moritake, and K. Toda, "Study of orientation of liquid crystal molecules at interface by shear horizontal wave," in Proceedings of the 14th IEEE International Conference on Dielectric Liquids (ICDL '02), pp. 386-389, July 2002.

[21] Y. Garbovskiy, L. Reisman, Z. Celinski, R. E. Camley, and A. Glushchenko, "Metallic surfaces as alignment layers for nondisplay applications of liquid crystals," Applied Physics Letters, vol. 98, no. 7, Article ID 073301, 2011.

[22] P. Yaghmaee, T. Kaufmann, B. Bates, and C. Fumeaux, "Effect of polyimide layers on the permittivity tuning range of liquid crystals," in Proceedings of the 6th European Conference on Antennas and Propagation, pp. 3579-3582, 2012.

[23] P. G. de Gennes, J. Prost, and R. Pelcovits, “The physics of liquid crystals," Physics Today, vol. 48, p. 70, 1995.

[24] A. Gaebler, A. Moessinger, F. Goelden et al., "Liquid crystalreconfigurable antenna concepts for space applications at microwave and millimeter waves," International Journal of Antennas and Propagation, vol. 2009, Article ID 876989, 7 pages, 2009.

[25] V. G. Chigrinov, Liquid Crystal Devices: Physics and Applications, Artech House, London, UK, 1999.

[26] G. Perez-Palomino, J. A. Encinar, and M. Barba, "Accurate electromagnetic modeling of liquid crystal cells for reconfigurable reflectarrays," in Proceedings of the 5th European Conference on Antennas and Propagation (EUCAP '11), pp. 997-1001, Rome, Italy, April 2011.

[27] D. Sievenpiper and J. Schaffner, "Beam steering microwave reflector based on electrically tunable impedance surface," Electronics Letters, vol. 38, no. 21, pp. 1237-1238, 2002.

[28] J. F. Bernigaud, N. Martin, P. Laurent et al., "Liquid crystal tunable filter based on DBR topology," in Proceedings of the 
36th European Microwave Conference (EuMC '06), pp. 368-371, Manchester, England, September 2006.

[29] W. Hu, M. Y. Ismail, R. Cahill et al., "Tunable liquid crystal reflectarray patch element," Electronics Letters, vol. 42, no. 9, pp. 509-511, 2006.

[30] N. Martin, P. Laurent, C. Person et al., "Influence of design liquid crystal-based devices on the agility capability," in Proceedings of the IEEE MTT-S International Microwave Symposium Digest, pp. 1835-1838, June 2005.

[31] M. Y. Ismail and R. Cahill, "Application of liquid crystal technology for electronically scanned reflectarrays," in Proceedings of the Asia-Pacific Conference on Applied Electromagnetics (APACE '05), pp. 82-85, December 2005.

[32] F. Goelden, A. Gaebler, O. Karabey, M. Goebel, A. Manabe, and R. Jakoby, "Tunable band-pass filter based on liquid crystal," in Proceedings of the German Microwave Conference (GeMIC '10), pp. 98-101, Berlin, Germany, March 2010.

[33] M. Yazdanpanahi and D. Mirshekar-Syahkal, "Millimeter-wave liquid-crystal-based tunable bandpass filter," in Proceedings of the IEEE Radio and Wireless Symposium (RWS '12), pp. 139-142, Santa Clara, Calif, USA, January 2012.

[34] P. Yaghmaee, A. K. Horestani, B. Bates, and C. Fumeaux, "A multi-layered tunable stepped-impedance resonator for liquid crystal characterization," in Proceedings of the Asia-Pacific Microwave Conference, pp. 776-778, 2012.

[35] D. Dolfi, M. Labeyrie, P. Joffre, and J. P. Huignard, "Liquid crystal microwave phase shifter," Electronics Letters, vol. 29, no. 10, pp. 926-928, 1993.

[36] C. Weil, G. Luessem, and R. Jakoby, "Tunable invertedmicrostrip phase shifter device using nematic liquid crystals," in Proceedings of the IEEE MTT-S International Microwave Symposium Digest, vol. 1, pp. 367-370, Seattle, Wash, USA, June 2002.

[37] F. Goelden, A. Gaebler, M. Goebel, A. Manabe, S. Mueller, and R. Jakoby, "Tunable liquid crystal phase shifter for microwave frequencies," Electronics Letters, vol. 45, no. 13, pp. 686-687, 2009.

[38] T. Kuki, H. Fujikake, and T. Nomoto, "Microwave variable delay line using dual-frequency switching-mode liquid crystal," IEEE Transactions on Microwave Theory and Techniques, vol. 50, no. 11, pp. 2604-2609, 2002.

[39] T. Kuki, H. Fujikake, H. Kamoda, and T. Nomoto, "Microwave variable delay line using a membrane impregnated with liquid crystal," in Proceedings of the IEEE MTT-S International Microwave Symposium Digest, vol. 1, pp. 363-366, Seattle, Wash, USA, June 2002.

[40] O. H. Karabey, A. Gaebler, S. Strunck, and R. Jakoby, "A 2-D electronically steered phased-array antenna with $2 \times 2$ elements in LC display Technology," IEEE Transactions on Microwave Theory and Techniques, vol. 60, no. 5, pp. 1297-1306, 2012.

[41] N. Martin, P. Laurent, C. Person, P. Gelin, and F. Huret, "Patch antenna adjustable in frequency using liquid crystal," in Proceedings of the 33rd European Microwave Conference, vol. 2, pp. 699-702, 2003.

[42] N. Martin, P. Laurent, C. Person, P. Gelin, and F. Huret, "Size reduction of a liquid crystal-based, frequency-adjustable patch antenna," in Proceedings of the 34th European Microwave Conference, vol. 2, pp. 825-828, Amsterdam, The Netherlands, October 2004.

[43] L. Liu and R. J. Langley, "Liquid crystal tunable microstrip patch antenna," Electronics Letters, vol. 44, no. 20, pp. 1179-1180, 2008.
[44] C. Fritzsch, S. Bildik, and R. Jakoby, "Ka-band frequency tunable patch antenna," in Proceedings of the IEEE Antennas and Propagation Society International Symposium, pp. 1-2, 2012.

[45] C. Damm, M. Maasch, R. Gonzalo, and R. Jakoby, “Tunable composite right/left-handed leaky wave antenna based on a rectangular waveguide using liquid crystals," in Proceedings of the IEEE MTT-S International Microwave Symposium (MTT '10), pp. 13-16, Anaheim, Calif, USA, May 2010.

[46] R. Marin, A. Mossinger, J. Freese, A. Manabe, and R. Jakoby, "Realization of $35 \mathrm{GHz}$ steerable reflectarray using highly anisotropic liquid crystal," in Proceedings of the IEEE Antennas and Propagation Society International Symposium, pp. 43074310, 2006.

[47] R. Marin, A. Mössinger, J. Freese, S. Müller, and R. Jakoby, "Basic investigations of $35 \mathrm{GHz}$ reflectarrays and tunable unitcells for beamsteering applications," in Proceedings of the 2nd European Radar Conference (EURAD '05), pp. 291-294, Paris, France, October 2005.

[48] W. Hu, M. Y. Ismail, R. Cahill et al., "Liquid-crystal-based reflectarray antenna with electronically switchable monopulse patterns," Electronics Letters, vol. 43, no. 14, pp. 744-745, 2007.

[49] A. Moessinger, R. Marin, J. Freese, S. Mueller, A. Manabe, and R. Jakoby, "Investigations on $77 \mathrm{GHZ}$ tunable reflectarray unit cells with liquid crystal," in Proceedings of the 1st European Conference on Antennas and Propagation (EuCAP '06), pp. 1-4, Nice, France, November 2006.

[50] G. Perez-Palomino, J. A. Encinar, M. Barba, and E. Carrasco, "Design and evaluation of multi-resonant unit cells based on liquid crystals for reconfigurable reflectarrays," IET Microwaves, Antennas and Propagation, vol. 6, no. 3, pp. 348-354, 2012.

[51] G. Perez-Palomino, P. Baine, R. Dickie et al., "Design and experimental validation of liquid crystal-based reconfigurable reflectarray elements with improved bandwidth in F-band," IEEE Transactions on Antennas and Propagation, vol. 61, pp. 1704-1713, 2013.

[52] W. Hu, R. Cahill, J. A. Encinar et al., "Design and measurement of reconfigurable millimeter wave reflectarray cells with nematic liquid crystal," IEEE Transactions on Antennas and Propagation, vol. 56, no. 10, pp. 3112-3117, 2008.

[53] O. H. Karabey, S. Bausch, S. Bildik, S. Strunck, A. Gaebler, and R. Jakoby, "Design and application of a liquid crystal varactor based tunable coupled line for polarization agile antennas," Proceedings of the 42nd European Microwave Conference, pp. 739-742, 2012.

[54] O. H. Karabey, S. Bildik, S. Bausch, S. Strunck, A. Gaebler, and R. Jakoby, "Continuously polarization agile antenna by using liquid crystal-based tunable variable delay lines," IEEE Transactions on Antennas and Propagation, vol. 61, pp. 70-76, 2013.

[55] P. Yaghmaee, W. Withayachumnankul, A. K. Horestani, A. Ebrahimi, B. Bates, and C. Fumeaux, "Tunable electric-LC resonators using liquid crystal," in Proceedings of the IEEE International Symposium on Antenna and Propagation (APS/USNC-URSI '13), 2013.

[56] W. Hu, R. Dickie, R. Cahill et al., "Liquid crystal tunable mm wave frequency selective surface," IEEE Microwave and Wireless Components Letters, vol. 17, no. 9, pp. 667-669, 2007.

[57] F. Zhang, Q. Zhao, L. Kang et al., "Magnetic control of negative permeability metamaterials based on Liquid Crystals," in Proceedings of the 38th European Microwave Conference (EuMC '08), pp. 801-804, Amsterdam, The Netherlands, October 2008. 
[58] J. A. Bossard, X. Liang, L. Li et al., “Tunable frequency selective surfaces and negative-zero-positive index metamaterials based on liquid crystals," IEEE Transactions on Antennas and Propagation, vol. 56, no. 5, pp. 1308-1320, 2008.

[59] I. C. Khoo, A. Diaz, J. Bossard, X. Liang, and D. Werner, "Nanodispersed liquid crystalline structures for tunable negative, zero-, and positive index materials in the optical-terahertz regimes," in Proceedings of the International Symposium on Biophotonics, Nanophotonics and Metamaterials (Metamaterials '06), pp. 412-416, Hangzhou, China, October 2006.

[60] J. A. Bossard, L. Li, D. H. Werner, and I. C. Khoo, "Infrared liquid crystal tunable frequency selective surfaces," in Proceedings of the IEEE Antennas and Propagation Society International Symposium, pp. 4489-4492, 2006.

[61] M. Hoefle, M. Koeberle, A. Penirschke, and R. Jakoby, "Improved millimeter wave Vivaldi antenna array element with high performance liquid crystals," in Proceedings of the 36th International Conference on Infrared, Millimeter, and Terahertz Waves (IRMMW-THz '11), pp. 1-2, Houston, Tex, USA, October 2011.

[62] S. Christie, R. Cahill, N. Mitchell, Y. Munro, and A. Manabe, "Liquid crystal based Rotman lens antenna with switchable monopulse patterns," Microwave and Optical Technology Letters, vol. 55, pp. 2721-2726, 2013. 

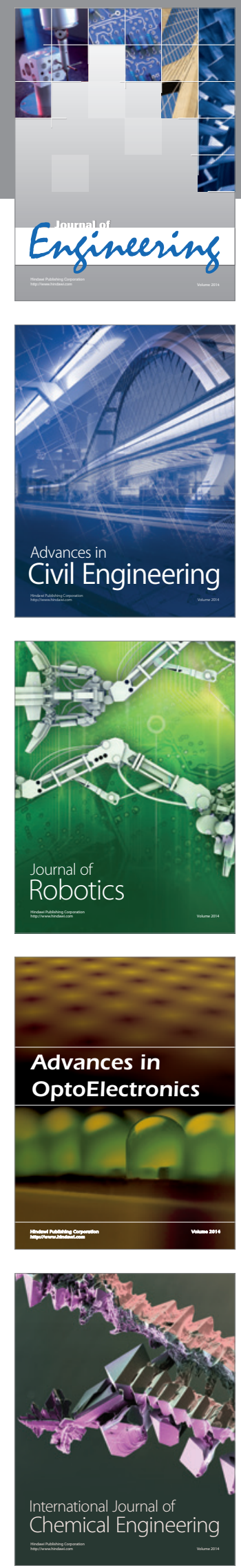

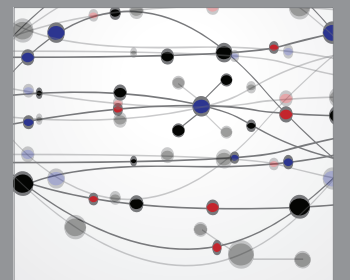

The Scientific World Journal
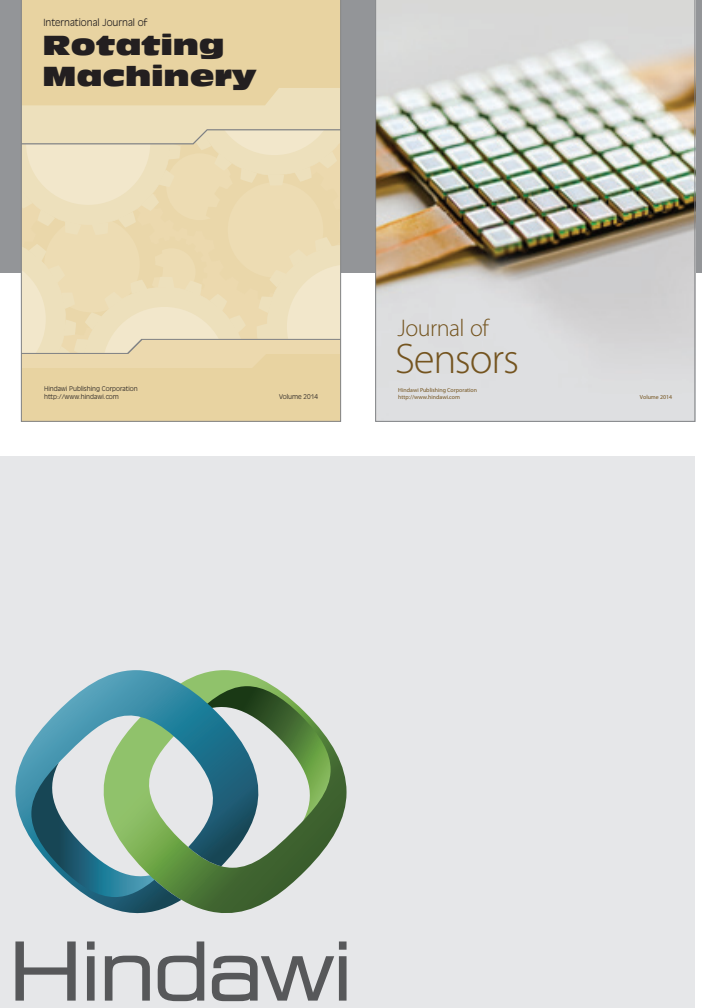

Submit your manuscripts at http://www.hindawi.com
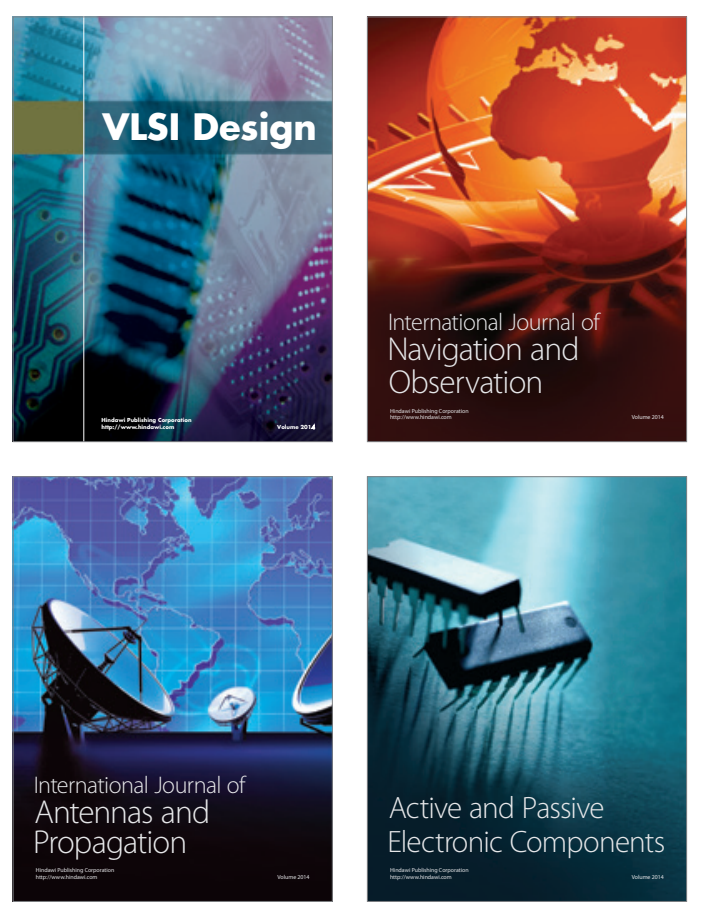
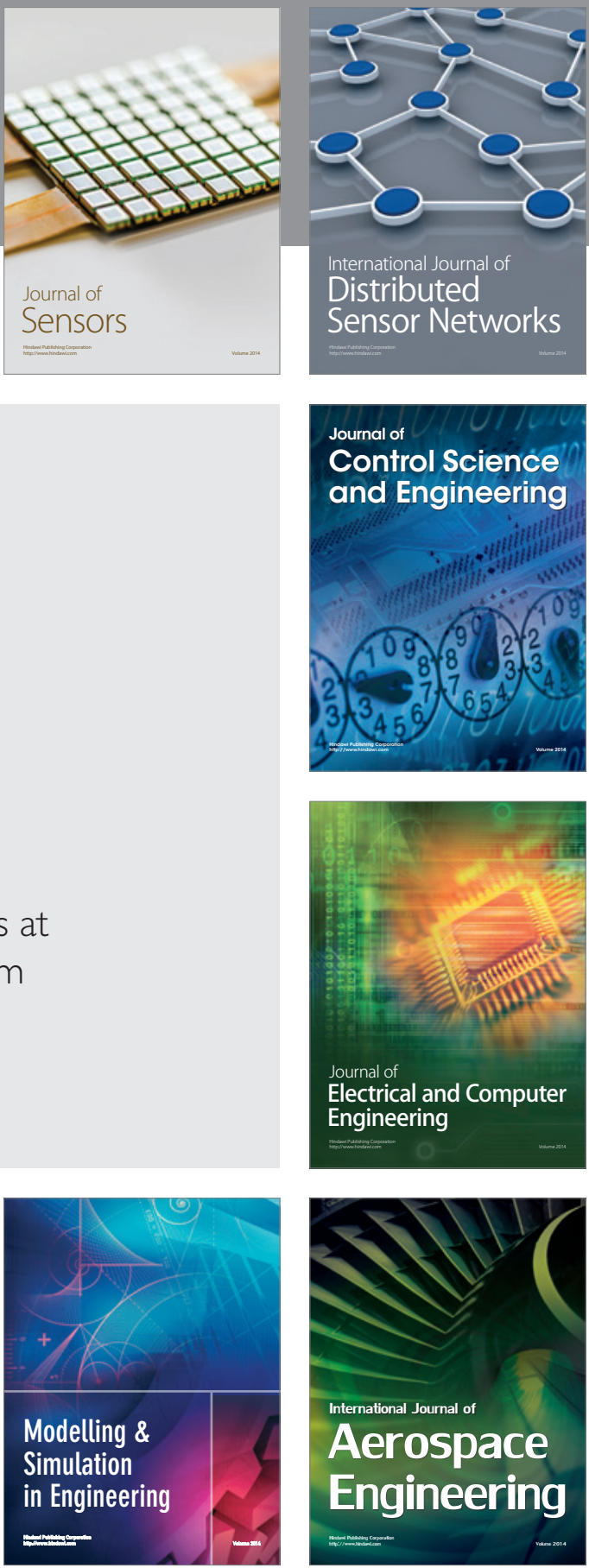

Journal of

Control Science

and Engineering
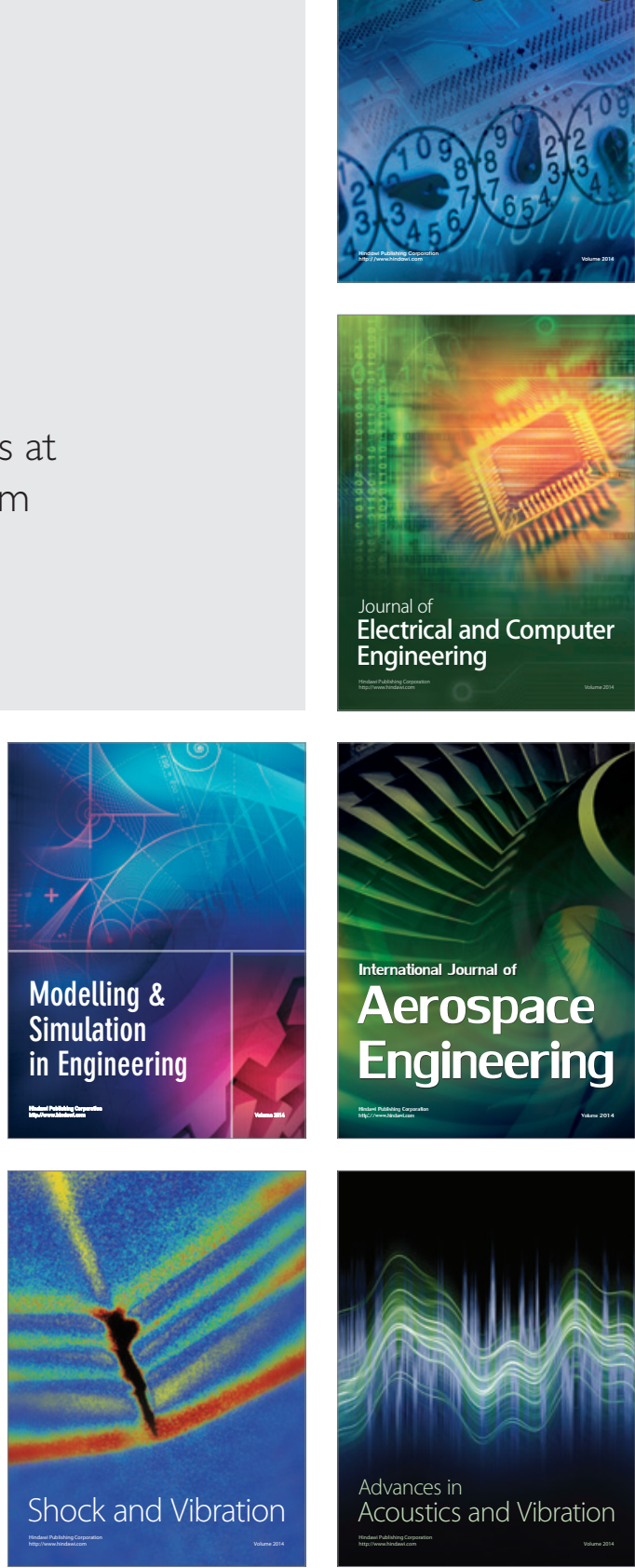